\title{
Performance Enhancement of Millimeter Wave Antenna with Integrated Inter-digital Capacitor Structure
}

\author{
Paritosh PESHWE, Ashwin KOTHARI \\ Dept. of Electronics and Communication Engineering, VNIT, Nagpur, India \\ paritoshpeshwe@gmail.com, ashwinkothari@ece.vnit.ac.in \\ Submitted August 18, 2017 / Accepted June 21, 2018
}

\begin{abstract}
In this paper, we present an approach for performance enhancement of an antenna for millimeter wave (mmWave) applications. From mmWave band, a $30 \mathrm{GHz}$ frequency is selected for designing a patch antenna as it is suitable for the number of applications. Then the designed antenna is modified by inserting a capacitive structure i.e., inter-digital capacitor (IDC) for better impedance matching. Insertion of IDC structure results in antenna size reduction along with the increased directivity and enhancement in gain. Similarly, this technique is capable of providing extra resonance with an advantage of fine tuning, as it offers control over both resonating frequencies independently. Further, a linear antenna array is designed with the same approach for mutual coupling analysis. From the results, it has been found that this technique is also capable of providing mutual coupling mitigation. Thus insertion of IDC structure provides overall performance enhancement in mmWave antenna.
\end{abstract}

\section{Keywords}

Millimeter wave antenna, dual-band characteristics, performance enhancement, capacitive loading

\section{Introduction}

Over the last decade, there have been conspicuous advances in the field of wireless communication, most of the wireless applications use ultra \& super high frequency bands. This escalating demand has resulted in saturation of the available bands thus leading to diminution on the available bandwidth as well as data rate. An approach to use the frequency bands which are unexploited and are available for various applications is highly desirable to overcome the aforesaid problem.

Millimeter wave (mmWave) technology works on the frequency band ranging from $30 \mathrm{GHz}$ to $300 \mathrm{GHz}$. For mmWave frequency, the size of the antenna required is very small thus resulting in smaller ranges for communication. To overcome this problem antenna must be used in an array so that overall gain can get increased along with the range [1]. Though mmWave frequency spectrum is unutilized, researchers are exploring it for various applications like body centric communication [2], detection of concealed threats [3] and 5G communication [4]. Above discussed applications require antenna with characteristics like

- Higher gain for high penetrating capabilities.

- Compact dimensions to have less mutual coupling.

- Narrow bandwidth.

- Highly directive for efficient area coverage.

- Multiband.

There are few techniques available in literature which improve any one of the above mentioned characteristics, like use of hybrid substrate or superstrate layer for antenna fabrication for gain enhancement [5]; use of various structures like electromagnetic band gap structures between the array elements for suppression of surface-wave for mutual coupling reduction [6]; approach of using parasitic shorting strips for antenna size reduction [7]. Further, there are few techniques which can simultaneously improve any two of the above characteristics like the use of a defected ground plane for mutual coupling reduction [8] and multiband resonance [9]. Similarly, metamaterial or composite right/left handed (CRLH) material is also considered for achieving improvement in two or more characteristics of the antenna. As mentioned in [10] metamaterial is used in an antenna for miniaturization and mutual coupling improvement; also in [11] size reduction along with multiband resonance is procured using metamaterial structure. Alternatively, a fractal tree structure is another approach by which miniaturization can be achieved along with the multiband characteristics [12].

All these techniques are difficult for fabrication and often impractical for a dedicated application. Similarly, implementation of any of these technologies results in improvement of either one or two characteristics of an antenna at a time. But, it is very challenging to improve all the above discussed characteristics of an antenna simultaneously. Hence in this paper, we propose an approach by which all the characteristics of an antenna are improved for achieving the application requirement. Similarly, after 
acquiring dual band characteristics by this approach, the resonances in a dual band can be altered individually. This unique capability of fine tuning makes designing of antenna flexible in terms of resonance and bandwidth.

The organization of this paper is as follows: Section 2 presents the design methodology and results of the patch antenna with and without IDC for $30 \mathrm{GHz}$. From the range of mmWave band, a $30 \mathrm{GHz}$ frequency is selected for the antenna design as it is suitable for the number of applications [2], [13]. Similarly discussion includes basics of inter-digital capacitor (IDC). Section 3 presents analysis of fine tuning after insertion of IDC structure on antenna. Section 4 gives comparison of results obtained from both the antenna designs along with the existing antenna in literature. Section 5 presents array analysis for verifying the mutual coupling between the antennas and finally conclusions from obtained results are drawn in Sec. 6. All antennas discussed in this paper are designed and simulated using HFSS software.

\section{IDC-based Antenna Design Methodology}

For designing of this antenna we have started with the designing of edge feed rectangular patch antenna as an initial stage and then same designed antenna is modified by loading inter-digital capacitor (IDC) structure in it.

\subsection{Patch Antenna Design}

The rectangular patch antenna is designed for the resonant frequency of $30 \mathrm{GHz}$ as an initial stage. The standard antenna equations mentioned in [14] are used for calculating the various dimension of the rectangular patch. Then, dimensions are optimized for impedance matching at the desired frequency. The substrate size is $15 \mathrm{~mm} \times 12 \mathrm{~mm} \times 0.8 \mathrm{~mm}$ with top surface consisting of a rectangular patch of dimensions $5.9 \mathrm{~mm} \times 3.25 \mathrm{~mm}$ and full ground plane at the bottom as shown in Fig. 1(a). The antenna is fed with a microstrip line of width $2.38 \mathrm{~mm}$ with edge feed of width $1.6 \mathrm{~mm}$ resulting in an impedance match of $50 \Omega$ and the substrate used is Rogers RT duroid 5880 with a dielectric constant $\varepsilon_{\mathrm{r}}=2.2$, loss tangent $(\tan \zeta)$ $=0.0009$ and copper thickness $=60 \mu \mathrm{m}$. Two hole panel mount $\mathrm{K}$ type connector is used to feed input signal which supports frequency range from DC to $40 \mathrm{GHz}$. The fabricated patch antenna design is shown in Fig. 1(b).

To check the impedance matching of the designed patch antenna, Smith chart has been plotted as shown in Fig. 1(c). Ideally, at the point of resonance, the impedance plot in Smith chart should touch its center i.e. $(1,0)$ location. We can conclude from Fig. 1(c) that the antenna terminal impedance is not matching properly as the plot is away from the real axis of the Smith chart. The impedance of an antenna at $30 \mathrm{GHz}$ comes out to be $48+11 \mathrm{j}$. To move the impedance plot further towards the real axis we

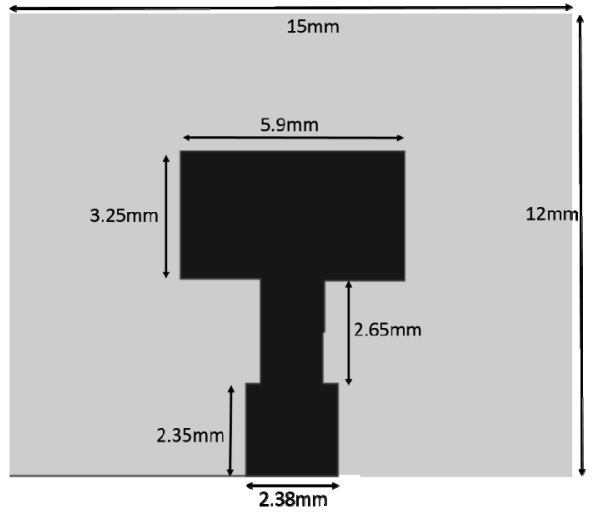

(a)

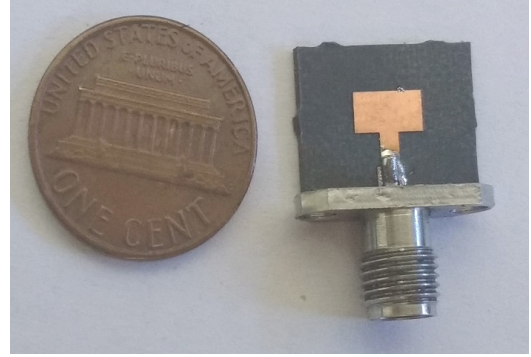

(b)

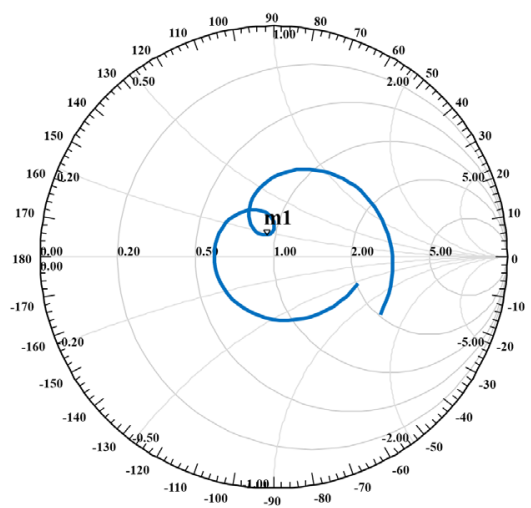

(c)

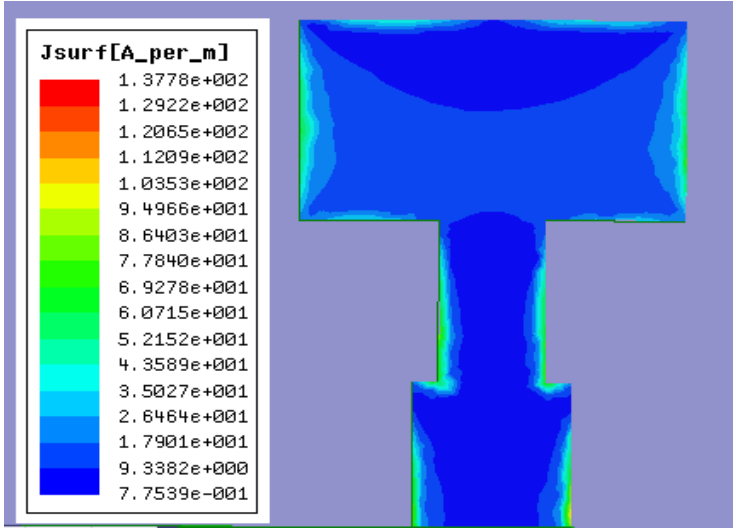

(d)

Fig. 1. (a) Designed patch antenna. (b) Fabricated patch antenna. (c) Smith chart of patch antenna with marker showing impedance at $30 \mathrm{GHz}$. (d) Surface current distribution at $30 \mathrm{GHz}$

must compensate the imaginary component of antenna impedance. As the reactance part in antenna impedance is positive, to compensate we must introduce a series capaci- 
tance into the antenna. Insertion of the inter-digital capacitor structure to feed line provides good impedance matching which results in the reduction in the size of an antenna [15]. So, similar IDC structure is inserted but onto the patch surface instead of the feed line.

\subsection{IDC Structure Basics}

The inter-digital capacitor (IDC) structure is a multifinger planer structure where the narrow gap between the fingers gives capacitance. The structure is designed in such a way that it can be accommodated in a small area. The observed value of quality factor in case of IDC is comparatively higher than that of MIM capacitor and gap capacitor. The IDC structure has four parameters i.e. the number of fingers $N$, width of the fingers $W$, gap between the consecutive fingers $G$ and length of the finger $L$. The capacitance generated due to the structure changes depending upon the value of these parameters. The value of capacitance is directly proportional to the width, length and number of fingers and inversely to the gap between consecutive fingers. Figure 2(a) shows the design of IDC having 10 fingers.

To obtain optimum finger width value for the IDC structure, an empirical analysis is performed. Figure 2(b) shows the effect of variation of finger width from $0.1 \mathrm{~mm}$

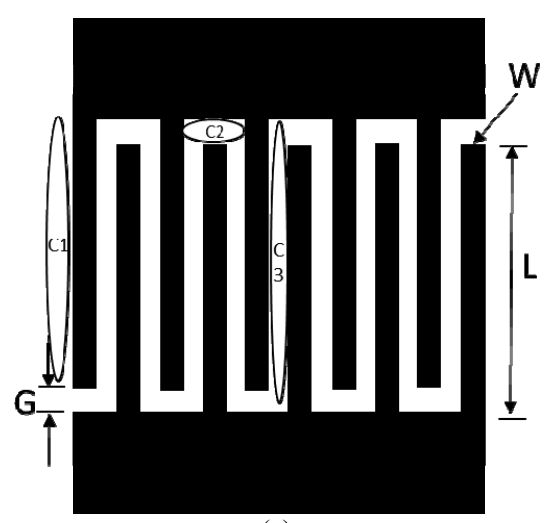

(a)

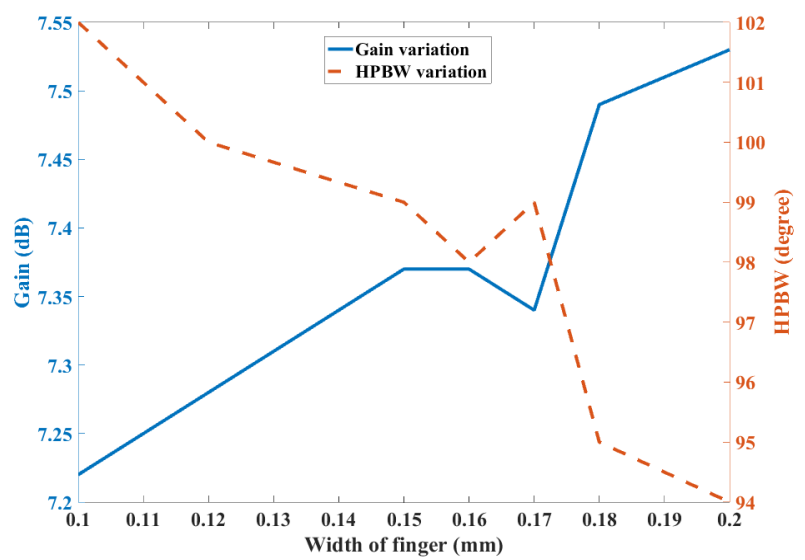

(b)

Fig. 2. (a) Interdigital capacitor structure. (b) Effect of variation of finger width with respect to peak gain and HPBW for $30 \mathrm{GHz}$ frequency. to $0.2 \mathrm{~mm}$ with respect to peak gain and half power beam width (HPBW). It is observed that the increase in the finger width results in an increment in peak gain while decrementing in HPBW. So for inserting the IDC structure, the optimized value for the width of the finger is considered as $0.15 \mathrm{~mm}$. Note that even though the value of peak gain is increasing with a finger width, the losses corresponding to an antenna are also increasing, which results in a lower value of radiation efficiency.

\subsection{Antenna Design using IDC}

The above mentioned IDC structure is inserted in the patch by creating a slot. After addition of the IDC structure, the response of the antenna was studied again; in which it has been found that the resonant frequency of the antenna has changed from $30 \mathrm{GHz}$ to $29.25 \mathrm{GHz}$. Hence to shift the resonant frequency back to $30 \mathrm{GHz}$ dimensions of the patch are altered. The patch dimensions for matching $30 \mathrm{GHz}$ resonance comes out to be $5.6 \mathrm{~mm} \times 3.1 \mathrm{~mm}$. The dimensions of the ground plane are $14 \mathrm{~mm} \times 11.7 \mathrm{~mm}$. All the dimensions of the designed patch antenna after insertion of IDC are shown in Fig. 3(a). It has been found that resonant frequency of the IDC depends on the combination of number of fingers, width of the finger and gap between the fingers. So, for better results in terms of reflection

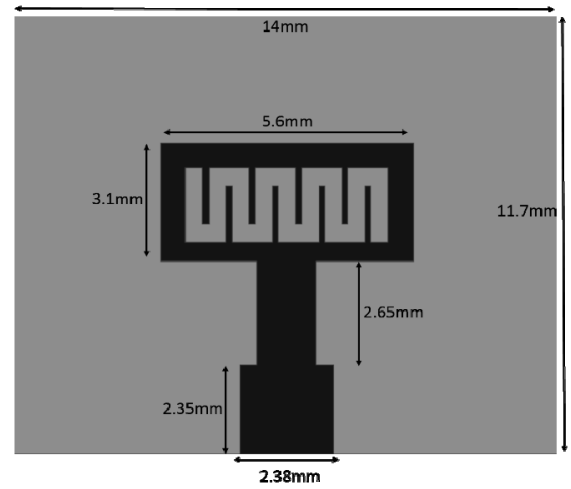

(a)

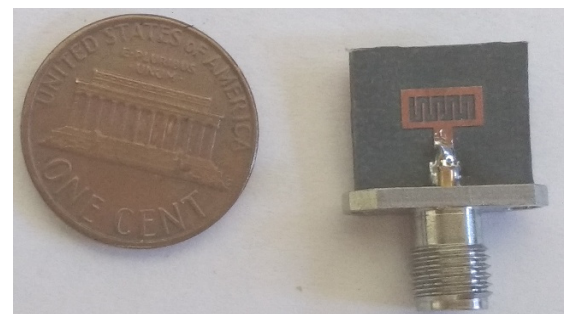

(b)

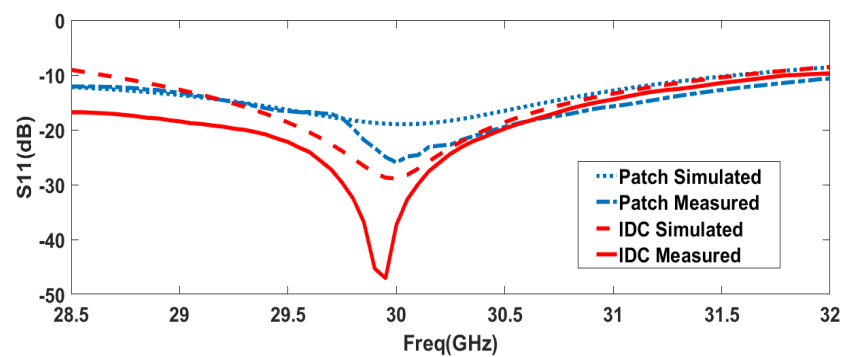

(c) 


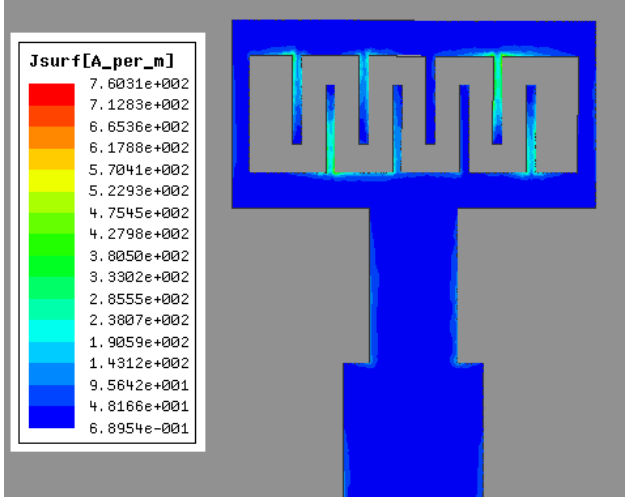

(d)

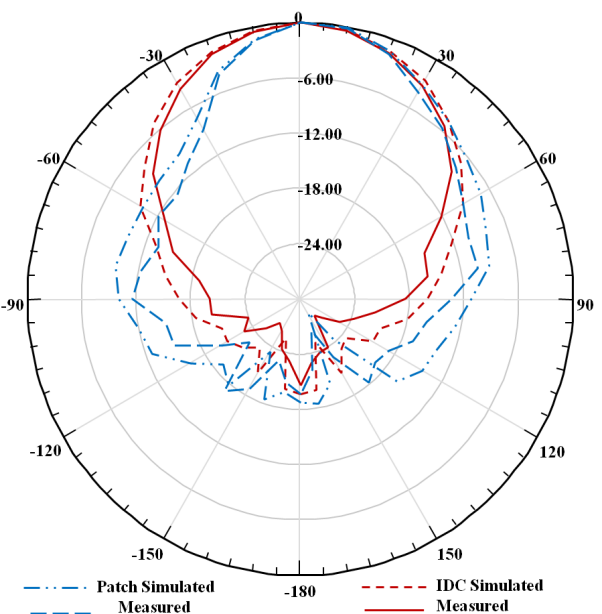

(e)

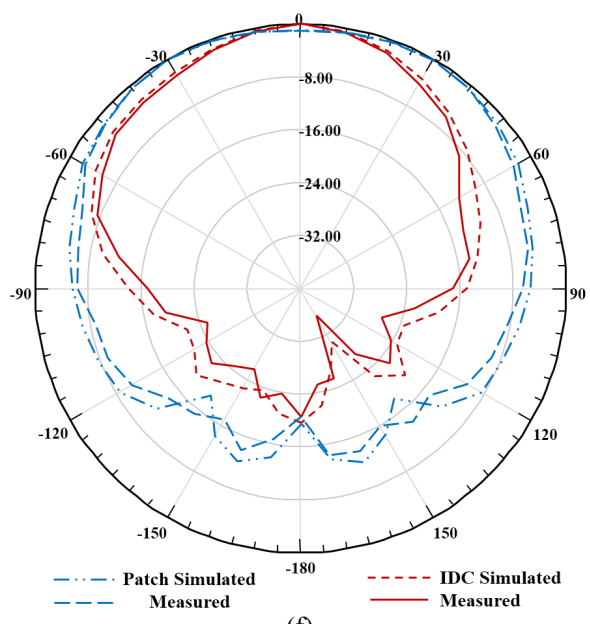

(f)

Fig. 3. (a) Designed patch antenna with IDC. (b) Fabricated IDC based patch antenna. (c) Reflection coefficient graph. (d) Surface current distribution at $30 \mathrm{GHz}$. (e) Measured and simulated 2D radiation pattern of patch antenna with and without IDC for $30 \mathrm{GHz}$ frequency in xz-plane $\left(\varphi=0^{\circ}\right)$ and (f) yz- plane $\left(\varphi=90^{\circ}\right)$.

coefficient at $30 \mathrm{GHz}$ frequency, values of IDC parameters come out to be $N=8$, with $W=0.15 \mathrm{~mm}, G=0.5 \mathrm{~mm}$ and $L=1.5 \mathrm{~mm}$.

Addition of IDC structure results in improvement of the reflection coefficient from $-19 \mathrm{~dB}$ to $-29 \mathrm{~dB}$ which signifies better matching of the antenna for the desired frequency. The fabricated antenna designed using IDC structure is shown in Fig. 3(b). Figure 3(c) shows the reflection coefficient response of simulated and measured results of the patch with and without IDC structure. It is observed that simulated and measured results are in good agreement. Surface current distribution of the designed antenna with IDC at $30 \mathrm{GHz}$ is shown in Fig. 3(d). Simulated and measured radiation pattern of the patch antenna with and without IDC in xz-plane and yz-plane can be observed from Fig. 3(e) and (f), respectively. We can conclude from Fig. 3(f) that the radiation pattern after insertion of IDC structure becomes narrower compared to the normal patch case which results in an increment of peak gain. Hence, the increased value of peak gain is due to the narrowing of the main lobe which makes energy radiated by an antenna more concentrated in the main lobe direction.

\subsection{Capacitive Effect in IDC}

The $\pi$ type transmission line model of IDC structure based antenna is shown in Fig. 4. The metal surface of patch and ground plane can be considered as a two wire transmission line model with the distributed series inductance $L_{\mathrm{R}}$ and distributed shunt capacitance $C_{\mathrm{R}}$ which are associated with the permeability and permittivity of the substrate respectively. Similarly, the dielectric conductivity of the substrate can be modelled as shunt inductance $L_{\mathrm{L}}$. Usually, the value of shunt inductance remains very small and can be neglected for few particular cases. Inserted IDC structure can be modelled as the series capacitor $C_{\mathrm{L}}$ along the patch. Other circuit components are not considered in this discussion as the value of those components depends on the substrate and patch properties which are same for both designs. For IDC structure, the capacitor $C_{\mathrm{L}}$ can be represented by the combination of three capacitances involved in the design. As shown in Fig. 2 (a) $C_{1}$ is the capacitance because of the two extreme fingers with the elements present on the outer side of the IDC along the length of the patch. $C_{2}$ is the capacitance because of the gap near the tip of the finger along the width of the patch. Similarly, $C_{3}$ is the capacitance along the length because of the two adjacent fingers. Hence, the overall value of the capacitance $C_{\mathrm{L}}$ because of the IDC structure having $N$ fingers can be given by:

$$
C_{L}=C_{1}+N C_{2}+(N-2) C_{3} .
$$

There are different approaches in the literature for calculation of $C_{1}, C_{2}$ and $C_{3}$. Similarly, there are few assumptions for optimization like mutual capacitance is con-

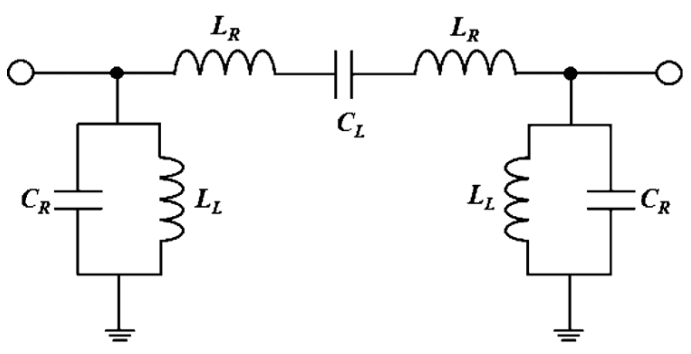

Fig. 4. $\pi$ type transmission line model of the design [16]. 
sidered in adjacent fingers only and capacitance $C_{2}$ is neglected due to the lower value, etc. The representation of interdigital capacitor structure is described with its corresponding subcomponents in [17]. The computation of capacitance value is essential for the practical realization of the structure and for deriving lumped equivalent circuit model.

\section{Analysis}

To understand the impact of insertion of IDC structure, an analysis is performed with respect to reflection coefficient. It has been observed that the insertion of IDC results in giving an extra resonance. If this resonance matches with the resonance generated by patch then there will be only one resonance corresponding to that antenna. But in all other cases, insertion of IDC will give an extra resonance. Initially, capacitance generated by the IDC structure has been changed by changing the width of the finger and keeping patch dimensions as it is. Figure 5(a) gives the variation of reflection coefficient by changing finger width $W$ gradually from $0.1 \mathrm{~mm}$ to $0.2 \mathrm{~mm}$. All other parameters are kept same throughout the analysis, patch length is kept as $3.4 \mathrm{~mm}, N=4, G=0.7 \mathrm{~mm}$ and $L=1.5 \mathrm{~mm}$. Here increment in the width of finger resulted in an increase of capacitance and the increased capacitance

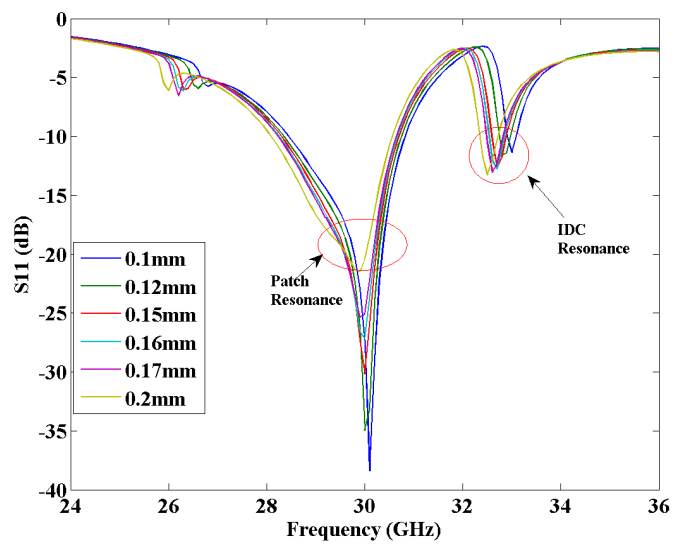

(a)

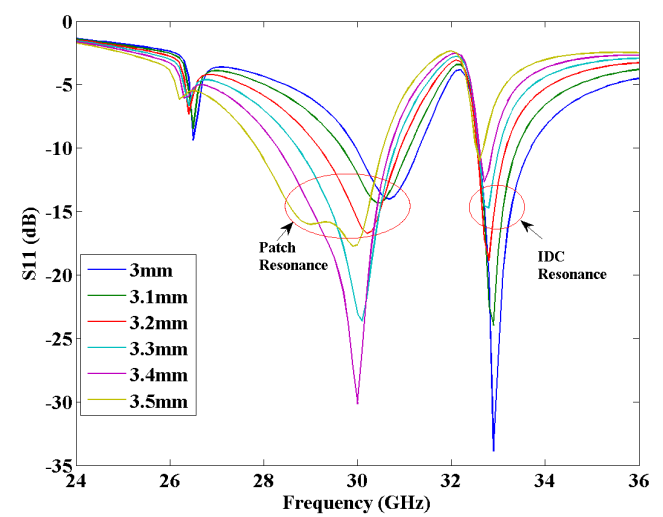

(b)

Fig. 5. (a) Variation in IDC structure keeping patch dimensions constant. (b) Variation in patch length keeping IDC structure constant. shifted the resonant frequency towards left. The variation in IDC structure results in the change of resonant frequency corresponding to IDC resonance while keeping the patch resonance as almost constant. To verify the above analysis, now the IDC structure has been kept constant with $N=4, G=0.7 \mathrm{~mm}, L=1.5 \mathrm{~mm}$ and $W=0.15 \mathrm{~mm}$ while the length of the patch has been varied from $3 \mathrm{~mm}$ to $3.5 \mathrm{~mm}$. In this case, patch resonance has changed while the IDC resonance is almost constant as shown in Fig. 5(b). To get single resonance at any desired frequency, the combination of IDC and patch length must be varied in such a way that overall generated capacitances give only one resonant peak.

The main challenge in designing dual band antenna is to get resonating bands exactly at the required frequencies. For various applications with dual band antenna, we can insert capacitive structure as it will provide additional resonance with an advantage of control over both the resonating frequencies. We can alter either of the resonating frequencies without changing the other, so this can be considered as tuning technique for matching resonances in the multiband antenna. This can be observed from Fig. 5(a) and Fig. 5(b) that two resonances can be varied by either changing IDC structure or changing the length of a patch. This multiband property can be used for designing a Kaband satellite communication antenna with an uplink of $28 \mathrm{GHz}-30 \mathrm{GHz}$ and downlink of $18 \mathrm{GHz}-20 \mathrm{GHz}$.

\section{Comparison of Results}

We can observe from Tab. 1 that insertion of IDC in patch antenna results in the reduction of patch dimensions along with the advantage of better matching. Usually, insertion of reactive structure on patch antenna increases the $\mathrm{Q}$ factor which results in bandwidth reduction. So we can observe from Tab. 1 that the bandwidth got reduced after insertion of IDC structure. It can be observed from Fig. 1(d) that the majority of the surface current flows through the boundary of the patch which results in a broader radiation pattern for the normal patch antenna. On the other hand, it can be observed from Fig. 3(d) that the majority of current

\begin{tabular}{|l|c|c|c|}
\hline Parameters & $\begin{array}{c}\text { Patch w/o } \\
\text { IDC }\end{array}$ & $\begin{array}{c}\text { Patch with } \\
\text { IDC }\end{array}$ & {$[18]$} \\
\hline $\begin{array}{l}\text { Antenna dimensions } \\
(\mathrm{mm})\end{array}$ & $15 \times 12$ & $14 \times 11.7$ & $19 \times 17$ \\
\hline $\begin{array}{l}\text { Patch dimensions } \\
(\mathrm{mm})\end{array}$ & $5.9 \times 3.25$ & $5.6 \times 3.1$ & $3.9 \times 3.24$ \\
\hline S11 at 30 GHz (dB) & $-19(-32)$ & $-29(-47)$ & $-32(-28)$ \\
\hline Peak gain $(\mathrm{dB})$ & $6.89(5.9)$ & $8(7.2)$ & 8.47 \\
\hline Bandwidth $(\mathrm{GHz})$ & $6(7.5)$ & $3(4.4)$ & $2(2)$ \\
\hline Fractional B/W $(\%)$ & $20(25)$ & $10(14.6)$ & $6.66(6.66)$ \\
\hline HPBW & $123^{\circ}\left(109^{\circ}\right)$ & $62^{\circ}\left(53^{\circ}\right)$ & - \\
\hline $\begin{array}{l}\text { Radiation efficiency } \\
(\%)\end{array}$ & 98.2 & 97.7 & 97.6 \\
\hline Dual band & No & Yes & No \\
\hline
\end{tabular}

Tab. 1. Result comparison of both antenna designs with metamaterial antenna designed in [18] for $30 \mathrm{GHz}$. All measured values are mentioned in the brackets. 


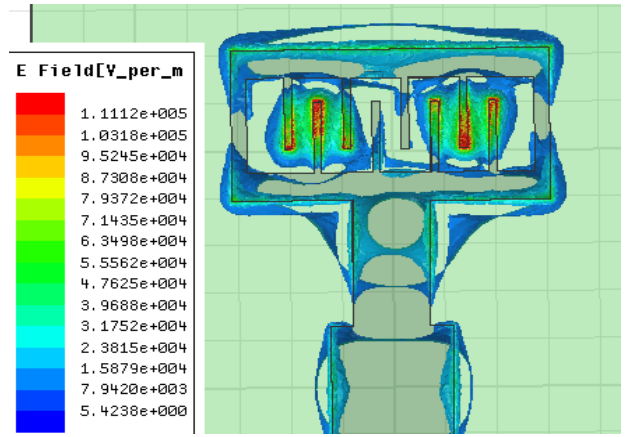

Fig. 6. E-field plot of patch antenna with IDC at $30 \mathrm{GHz}$ frequency.

flows through the fingers of IDC. So we can conclude that the surface current flowing through patch gets confined within an IDC structure which results in more directive radiation pattern. This can also be verified from the values of HPBW of both the antennas mentioned in Tab. 1. The HPBW of patch antenna with IDC structure is much lesser than the normal patch. Similarly, insertion of IDC is responsible for generation of stronger fringing fields through the fingers as shown in Fig. 6. It can be observed from Fig. 6 that the intensity of field generated due to fingers is much higher than the rest of the patch. This in response increases flux density in that region and makes energy radiated by an antenna more concentrated which results in the enhancement of peak gain.

Similarly, we can observe that the radiation efficiency of the patch antenna with IDC is slightly reduced than that of the patch without IDC. The slight reduction in radiation efficiency is mainly due to the losses induced due to high intensity currents flowing through the fingers. This can also be observed in Fig. 3(d) that the intensity of the current flowing through the fingers is higher in comparison to the current flowing throughout the patch. This finger current increases the leakage current through the substrate of an antenna. Similarly, from a comparison of IDC loaded patch antenna with metamaterial antenna mentioned in [18], we can observe that in most of the characteristics, the proposed antenna outperformed the performance of antenna mentioned in the literature.

\section{Array Analysis}

In millimeter wave applications, use of single antenna is impractical as small wavelength results in the smaller range for communication. Hence most of the applications will need an array of an antenna where mutual coupling will play a vital role in overall array performance. To understand the impact of capacitive structure on mutual coupling, a two element linear array has been formed on Rogers RT duroid 5880 substrate using patch antenna with and without IDC as shown in Fig. 7(a) and Fig. 7(c). Figures 7(b) and (d) show fabricated linear antenna arrays. For the analysis distance between the patch elements of an array is kept as $\lambda_{0} / 4$ for both the cases. Simulated and measured mutual coupling results in both the arrays are

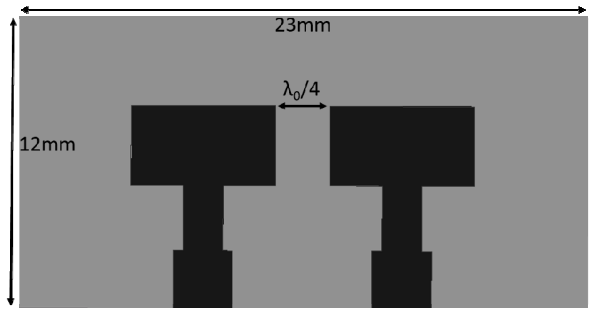

(a)

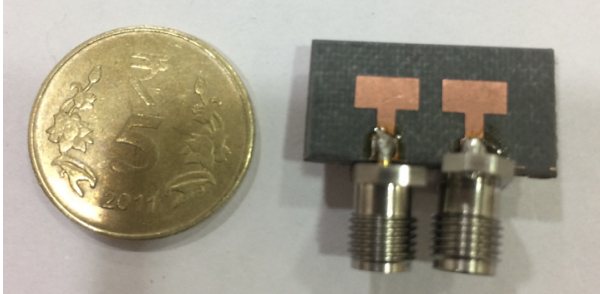

(b)

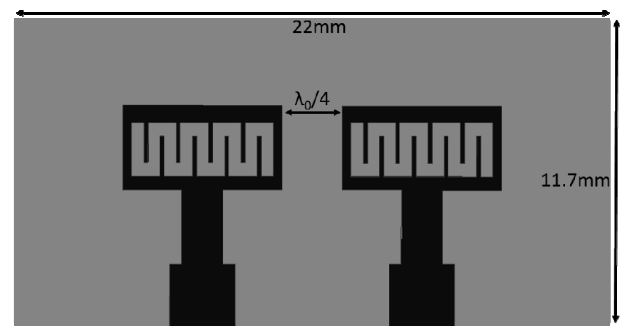

(c)

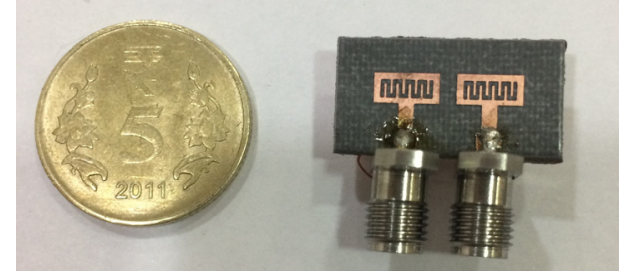

(d)

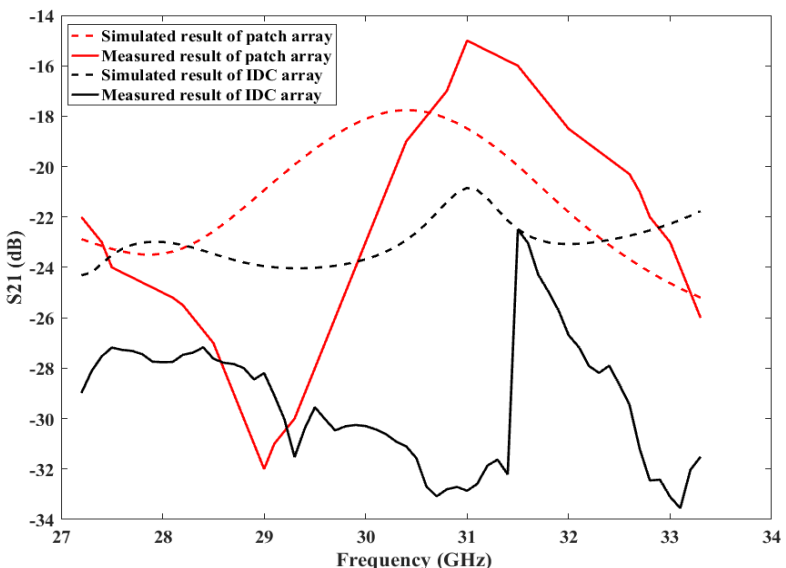

(e)

Fig. 7. (a) Designed two element linear array of patch antenna without IDC. (b) Fabricated two element linear array of patch antenna without IDC. (c) Designed array with IDC structure. (d) Fabricated array with IDC structure. (e) Measured and simulated mutual coupling between antenna array with and without IDC structure.

shown in Fig. 7(e). Mutual coupling in patch array without IDC comes out to be $-17 \mathrm{~dB}$. However, for a good isolated antenna array, the acceptable value for mutual coupling 
should be around $-16 \mathrm{~dB}$. So mutual coupling of $-17 \mathrm{~dB}$ is marginally better than the standard value. In patch antenna array with IDC, mutual coupling is improved from $-17 \mathrm{~dB}$ to $-25 \mathrm{~dB}$. This value is acceptable according to isolation terms. This improvement in mutual coupling between the elements of an array is because of inserted IDC structure, which permits more number of radiating elements on the same substrate. The variations in measured and simulated results are due to the errors introduced while fabrication, soldering and measurement.

\section{Conclusion}

In this paper, an approach was proposed for enhancing the overall performance of an antenna by loading IDC structure on it. After insertion of IDC, better impedance matching was observed which was also verified from the reflection coefficient graph. It also resulted in the reduction of patch dimensions by around $10 \%$ along with the conversion of a wideband antenna to narrowband. This approach had also provided an additional resonance with an advantage of fine tuning as we had control over both the resonating frequencies independently. Insertion of capacitive structure helped in giving directionality to an antenna, hence for applications requiring a high directive antenna, this can be beneficial. Similarly, array analysis verified that the IDC patch resulted in the lower value of mutual coupling. Thus we can clearly state that the insertion of capacitive structure like IDC was responsible for the reduction of patch dimensions along with providing higher gain and multiband characteristics.

\section{References}

[1] WANG, H., FANG, D. G., LIU, Y. K., et al. A millimeter wave cylindrical conformal array with conical beam. In Asia Pacific Microwave Conference (APMC). Singapore, 2009, p. 2010-2013. DOI: $10.1109 /$ APMC.2009.5385301

[2] PELlEGRINI, A., BRIZZI, A., ZHANG, L., et al. Antennas and propagation for body-centric wireless communications at millimeter-wave frequencies: A review [Wireless corner]. IEEE Antennas and Propagation Magazine, 2013, vol. 55, no. 4, p. 262-287. DOI: 10.1109/MAP.2013.6645205

[3] KEMP, M. C. A review of millimetre-wave and terahertz technology for detection of concealed threats. In $33^{\text {rd }}$ International Conference on Infrared, Millimeter and Terahertz Waves (IRMMW-THz 2008). Pasadena (CA, USA), 2008, p. 1-2. DOI: 10.1109/ICIMW.2008.4665630

[4] CAO, Z., MA, Q., SMOLDERS, A. B., et al. Advanced integration techniques on broadband millimeter-wave beam steering for $5 \mathrm{G}$ wireless networks and beyond. IEEE Journal of Quantum Electronics, 2016, vol. 52, no. 1, p. 1-20. DOI: 10.1109/JQE.2015.2509256

[5] ATTIA, H., YOUSEFI, L., BAIT-SUWAILAM, M. M., et al. Enhanced-gain microstrip antenna using engineered magnetic superstrates. IEEE Antennas and Wireless Propagation Letters. 2009; vol. 8, p. 1198-1201. DOI: 10.1109/LAWP.2009.2035149

[6] YANG, F., RAHMAT-SAMII, Y. Microstrip antennas integrated with electromagnetic band-gap (EBG) structures: A low mutual coupling design for array applications. IEEE Transactions on Antennas and Propagation, 2003, vol. 51, no. 10, p. 2936-2946. DOI: 10.1109 /TAP.2003.817983

[7] SO, KWOK KAN, WONG, H., LUK, K. M., et al. Miniaturized circularly polarized patch antenna with low back radiation for GPS satellite communications. IEEE Transactions on Antennas and Propagation, 2015, vol. 63, no. 12, p. 5934-5938. DOI: 10.1109/TAP.2015.2488000

[8] ZHU, F. G., XU, J. D., XU, Q. Reduction of mutual coupling between closely-packed antenna elements using defected ground structure. Electronics Letters, 2009, vol. 45, no. 12, p. 601-602. DOI: $10.1049 / \mathrm{el} .2009 .0985$

[9] PEI, J., WANG, A. G., GAO, S., et al. Miniaturized triple-band antenna with a defected ground plane for WLAN/WiMAX applications. IEEE Antennas and Wireless Propagation Letters, 2011, vol. 10, p. 298-301. DOI: 10.1109/LAWP.2011.2140090

[10] ABDALLA, M. A., IBRAHIM, A. A. Compact and closely spaced metamaterial MIMO antenna with high isolation for wireless applications. IEEE Antennas and Wireless Propagation Letters, 2013, vol. 12, p. 1452-1455. DOI: 10.1109/LAWP.2013.2288338

[11] AMANI, N., KAMYAB, M., JAFARGHOLI, A., et al. Compact tri-band metamaterial-inspired antenna based on CRLH resonant structures. Electronics Letters, 2014, vol. 50, no. 12, p. 847-848. DOI: 10.1049/el.2014.0875

[12] SRIVATSUN, G., SUBHA RANI, S. Compact multiband planar fractal cantor antenna for wireless applications: an approach. International Journal of Antennas and Propagation, 2012, p. 1-6. DOI: $10.1155 / 2012 / 839520$

[13] GHASR, M. T, KHARKOVSKY, S., BOHNERT, R., et al. $30 \mathrm{GHz}$ linear high-resolution and rapid millimeter wave imaging system for NDE. IEEE Transactions on Antennas and Propagation, 2013 vol. 61, no. 9, p. 4733-4740. DOI: 10.1109/TAP.2013.2270174

[14] BALANIS, C. Antenna Theory Analysis and Design. John Wiley \& Sons, 2005. ISBN-13: 978-0471667827

[15] LIU, J. X., YIN, W. Y. A compact interdigital capacitor-inserted multiband antenna for wireless communication applications. IEEE Antennas and Wireless Propagation Letters, 2010, vol. 9, p. 922-925. DOI: 10.1109/LAWP.2010.2073435

[16] SAM, S., KANG, H., LIM, S. Frequency reconfigurable and miniaturized substrate integrated waveguide interdigital capacitor (SIW-IDC) antenna. IEEE Transactions on Antennas and Propagation, 2014, vol. 62, no. 3, p. 1039-1045. DOI: 10.1109/TAP.2013.2281662

[17] BAHL, I. Lumped Elements for $R F$ and Microwave Circuits. Artech House, 2003, p. 229-250. ISBN 1-58053-309-4

[18] NECIBI, O., HAMZAOUI, D., VUONG, T. P., et al. A novel RFID-HIS-PRS reader antenna for the millimeter wave band $30 \mathrm{GHz}$. Microwave and Optical Technology Letters, 2015, vol. 57 , no. 8, p. 1835-1842. DOI: 10.1002/mop.29201

\section{About the Authors ...}

Paritosh D. PESHWE received B.E. degree in Electronics and Telecommunication Engineering in 2010. He further obtained M.E. degree in Microelectronics from Birla Institute of Technology \& Science, Pilani - Goa, India in 2013. He is currently pursuing Ph.D. degree from Visvesvaraya National Institute of Technology, Nagpur, India. His research areas include antenna design, antenna array 
optimization, metamaterials and millimeter-wave communication.

Ashwin KOTHARI received B.E. and M. Tech. degrees in Electronics Engineering in 1994 and 2005. He obtained Ph.D. degree from Visvesvaraya National Inst. of Technology, Nagpur, India in 2010, where he is currently working as an Associate Professor. He is one of the coordinators for Center of Excellence of COMMBEDDED SYSTEMS: Hybridization of Communications and Embedded Systems under World Bank assisted project of TEQIP 1.2.1. His research areas include antennas and wave propagation, electromagnetics, communication and Rough Sets. 